\title{
Review
}

nephron

Clinical

Practice

Published online: September 24, 2014

DOI: $10.1159 / 000363725$

\section{Clinical Trial Endpoints in Acute Kidney Injury}

\author{
Frederic T. Billings IV Andrew D. Shaw \\ Department of Anesthesiology, Vanderbilt University School of Medicine, Nashville, Tenn., USA
}

\section{Key Words}

Acute kidney injury - Chronic kidney disease - Clinical trials . Dialysis · Epidemiology · Intensive care unit - Major adverse kidney events

\begin{abstract}
The development and use of consensus criteria for acute kidney injury (AKI) diagnosis and the inclusion of recently identified markers of renal parenchymal damage as endpoints in clinical trials have improved the ability of physicians to compare the incidence and severity of AKI across patient populations, provided targets for testing new treatments, and may increase insight into the mechanisms of AKI. To date, these markers have not consistently translated into important clinical outcomes. Is that because these markers of renal injury/ dysfunction are measurements of process of care (and not indicative of persistently impaired renal function), or is it because patients do actually recover from AKI? Physicians currently have limited ability to measure renal function reserve, and the ultimate consequence of a case of AKI on long-term morbidity remains unclear. There is little doubt that groups of patients who develop AKI have worse outcomes than groups of patients who do not, but investigators are now realizing the value of measuring clinically meaningful renal endpoints in all subjects enrolled in AKI clinical trials. Impor-
\end{abstract}

tant examples of these outcomes include persistently impaired renal function, new hemodialysis, and death. We propose that these major adverse kidney events (MAKE) be included in all effectiveness clinical trials. Adaptation of the MAKE composite assessed 30,60, or 90 days following AKI (i.e., MAKE30 or MAKE90) will improve our capacity to understand and treat AKI and may also provide a consensus composite to allow comparison of different interventions. Primary endpoints for phase I and II clinical trials, on the other hand, should continue to use continuous markers of renal injury/dysfunction as well as 'hard' clinical outcomes in order to generate meaningful data with limited subject exposure to untested treatments. By doing so, investigators may assess safety without requiring large sample sizes, demonstrate treatment effect of an unknown therapeutic, and power subsequent studies. In contrast, phase III trials should include consensus AKI criteria and more important subsequent clinical outcomes, such as MAKE90, as primary endpoints.

$$
\text { (c) } 2014 \text { S. Karger AG, Basel }
$$

Targeting Recovery from Acute Kidney Injury: Round Table Conference at the 19th International Conference on Continuous Renal Replacement Therapies (Manchester Grand Hyatt, San Diego, Calif., USA, March 2-3, 2014).

\section{KARGER}

E-Mail karger@karger.com

www.karger.com/nec
(C) 2014 S. Karger AG, Basel

1660-2110/14/1274-0089\$39.50/0
Frederic T. Billings IV, MD, MSc

121121 st Avenue South, Suite 526

Nashville, TN 37212 (USA) 


\section{Introduction}

Endpoints for acute kidney injury (AKI) clinical trials include creatinine- and urine output-based criteria for AKI diagnosis, continuous scale- or threshold-based measurements of renal parenchymal damage, markers of renal filtration and solute elimination, requirement for renal replacement therapy, persistent decline in estimated glomerular filtration rate (eGFR), onset of chronic kidney disease (CKD), progression of CKD, and death. The objective of each trial influences the choice of endpoints. Diagnostic studies, biomarker validation studies, efficacy clinical trials, effectiveness clinical trials, risk prediction studies, prognostication studies, and safety studies carry different objectives. Selecting the appropriate renal endpoint is important. This paper will build the case that choosing a composite endpoint that includes death, newonset dialysis, and persistent renal disability is essential for high-impact effectiveness clinical trials (phase III clinical trials) and discusses why the AKI community should perhaps design studies that both measure the effects of treatments on acute pathophysiology and ultimate clinical outcomes.

\section{What Is AKI and Why Is It Important?}

An abrupt decline in kidney filtration of solutes, excretion of toxins, or resorption of electrolytes and water defines AKI. Renal glomerular, endothelial, or tubular injuries dictate these functional changes. The injured kidney is unable to clear toxic metabolites, including urea and fixed acids, or maintain volume and electrolyte homeostasis. Toxins and inflammatory mediators impair the function of extrarenal organs, and AKI is associated with subsequent infectious (wound infection and sepsis following major surgery), neurologic (delirium), and cardiac (myocardial infarction and atrial fibrillation) morbidity [1]. These extrarenal effects of AKI may explain the clinical observations that mild forms of AKI ( 0.3 or 0.5 $\mathrm{mg} / \mathrm{dl}$ increases in serum creatinine) increase the risk of extrarenal organ failure and are associated with a 7 -fold increase in 30-day mortality [2, 3]. In its more severe form, AKI requires renal replacement therapy, either continuous or intermittent hemodialysis. Hemodialysis is independently associated with a 50\% incidence of death among critically ill patients [4]. Persistent renal dysfunction complicates survival in a substantial number of the remaining patients and increases the mortality risk. Even when AKI is mild and serum creatinine concentrations return to baseline levels, AKI predisposes patients to subsequent CKD and increases the risk of subsequent AKI events and the risk of death [5]. It is indeed possible that 'total recovery' from AKI may not even exist, in large part because we do not have a way to measure renal function reserve or the extent to which an AKI episode compromises that reserve.

CKD is persistent renal dysfunction defined by a reduction in glomerular filtration. CKD is divided into 5 stages based on the eGFR. Stage $1 \mathrm{CKD}$ is an eGFR $>90$ $\mathrm{ml} / \mathrm{min} / 1.73 \mathrm{~m}^{2}$, stage 2 is $60-90$, stage 3 is $30-60$, stage 4 is $15-30$, and stage 5 is $<15 \mathrm{ml} / \mathrm{min} / 1.73 \mathrm{~m}^{2}$ or chronic renal replacement therapy (hemo- or peritoneal dialysis). The CKD Epidemiology Collaboration formula provides the best eGFR, although the modified diet in renal disease formula is also fairly robust, particularly for patients with significant CKD (stage 3 or worse). The hazard ratio for CKD progression following an acute episode of renal dysfunction ranges from 2.3 to 28.1 , depending on the clinical setting of AKI and AKI diagnostic criteria [6]. Severe CKD (stage 4 or 5) has devastating consequences. It is associated with a marked increase in the risk of myocardial infarction, sudden cardiac death, infection, immobility, and death [7]. The morbidity associated with CKD implores us to measure effectiveness of novel therapeutics for the prevention and salvage of AKI.

\section{How Do We Measure Renal Endpoints, and Why Should We Be Consistent?}

Injured glomeruli and tubules release compounds into the blood and urine, and we believe that quantification of these compounds should reflect the degree of injury - these are the 'damage biomarkers' of AKI, and their discovery has dominated the literature for the past 10 years. The ongoing identification and validation of novel AKI biomarkers has improved the understanding of AKI pathophysiology, time course, and recovery $[8,9]$. Since biomarkers released directly by injured renal parenchyma rise more quickly in the blood and urine than biomarkers cleared by the kidneys, measurement of injury biomarkers can provide an opportunity to enroll patients into AKI therapeutic trials. By inference, therefore, the identification of compounds in the blood or urine that are required for renal parenchymal repair may indicate AKI recovery. Proteomic analyses are providing opportunities to better characterize the initiation, propagation, resolution, and repair of AKI. We believe the identification and measurement of repair markers may better
Billings IV/Shaw 
Table 1. The relative benefit of endpoints of AKI clinical trials in regard to ease and cost of measurement, early expression, mechanistic insight, association with long-term morbidity, and clinician comfort in their assessment

\begin{tabular}{|c|c|c|c|c|c|c|}
\hline Endpoint & $\begin{array}{l}\text { Ease of } \\
\text { measure- } \\
\text { ment }\end{array}$ & $\begin{array}{l}\text { Early } \\
\text { expression }\end{array}$ & $\begin{array}{l}\text { Mechanism } \\
\text { insight }\end{array}$ & $\begin{array}{l}\text { Long-term } \\
\text { morbidity } \\
\text { association }\end{array}$ & $\begin{array}{l}\text { Low cost of } \\
\text { measurement }\end{array}$ & $\begin{array}{l}\text { Clinician } \\
\text { comfort in } \\
\text { assessment }\end{array}$ \\
\hline RIFLE, AKIN, KDIGO serum creatinine AKI criteria & $* * * *$ & $* *$ & $*$ & $* *$ & $* * * *$ & $* * *$ \\
\hline Renal parenchymal damage biomarkers & * & $* * * * *$ & $* * * *$ & $*$ & $*$ & $* *$ \\
\hline Renal function (clearance) biomarkers & * & $* * * *$ & * & $*$ & $*$ & $* *$ \\
\hline Persistent renal function decline ( $>25 \%$ eGFR reduction) & $* * *$ & * & * & $* * * *$ & $* * * *$ & $* * * *$ \\
\hline CKD progression (from stage 3 or 4 to stage 4 or 5 ) & $* * *$ & $*$ & * & $* * * *$ & $* * * *$ & $* * * *$ \\
\hline Death & $* * * * *$ & $*$ & $*$ & $* * * * *$ & $* * * * *$ & $* * * * *$ \\
\hline MAKE30 & $* * *$ & $*$ & $*$ & $* * *$ & $* * * *$ & $* * *$ \\
\hline MAKE60 & $* * *$ & $*$ & $*$ & $* * * *$ & $* * * *$ & $* * *$ \\
\hline
\end{tabular}

$*=$ Least benefit ${ }^{* * * * *}=$ greatest benefit; NA $=$ not applicable. MAKE (major adverse kidney events) is a composite of persistent renal function decline ( $>25 \%$ decline in eGFR), new requirement for hemodialysis, and death. MAKE is assessed 30, 60, or 90 days following AKI diagnosis.

indicate AKI recovery than the resolution of damage markers.

The common endpoint of impaired kidney function is reduced solute clearance from the blood, and serum quantification of 'function' markers such as creatinine and cystatin $\mathrm{C}$ reflects renal dysfunction. The delay in the creatinine rise following an AKI-inciting event, the lack of specificity due to extrarenal influences on serum concentrations, and the insensitivity to mild tubular injury limits the value of serum creatinine to assess AKI. Until more novel biomarkers are better characterized, however, the ubiquitous measurement of serum creatinine and the direct relationship between serum creatinine and glomerular filtration will continue to support the use of creatinine measurement to quantify AKI. Physician scientists and clinicians developed the Risk, Injury, Failure, Loss of Kidney Function, and End-Stage Renal Disease (RIFLE), Acute Kidney Injury Network (AKIN), and Kidney Disease: Improving Global Outcomes (KDIGO) criteria for AKI staging to address the pervasive use of highly heterogeneous creatinine threshold AKI diagnostic criteria in clinical studies and to provide a more sensitive indicator of clinically significant renal injury than the need for dialysis [10-12]. The use of these consensus criteria for AKI diagnosis, which exclusively use serum creatinine and urine output to stage AKI, has improved the ability of physicians to compare AKI across institutions and to better measure the relative efficacy of different therapies.
The development of consistent endpoints facilitates drug development, physician capacity to assess treatments, and benefits to patients - the ultimate stakeholders. Similar consensus should be developed for the assessment of persistent renal dysfunction, given the association of AKI with $\mathrm{CKD}$ and the morbidity dictated by CKD.

\section{How Do We Implement Meaningful Endpoints into AKI Clinical Trials?}

Endpoints of AKI clinical studies should be tailored to the type of investigation. Diagnostic investigation may require continuous markers of renal injury to best correlate the expression of novel biomarkers with AKI severity. Risk prediction and prognosis investigation may require the use of consensus criteria for AKI diagnosis and staging in order to establish the risk of subsequent renal and extrarenal disease and to define prognosis in terms of recovery or subsequent demise.

Primary endpoints in AKI clinical trials should include objective measurements of both the process of renal injury/dysfunction and the attendant ultimate clinical outcomes (table 1). AKI defined using RIFLE, AKIN, or KDIGO criteria (i.e., relative and absolute changes in serum creatinine or urine output over specific time intervals) and biomarkers of cell-specific injury or dysfunction constitute markers of renal injury and dysfunction. These 
endpoints are process-of-care measures that often indicate pathophysiology but may or may not consistently translate into significant clinical outcomes. The imperfect association between an episode of AKI and the development of clinically significant CKD supports the inclusion of hard outcomes in clinical trials. We should judge therapies based on real events rather than process-of-care measurements. For these reasons markers of renal injury/ dysfunction may not be the best primary endpoints for clinical trials but do contain specific advantages over more permanent and disabling renal outcomes. These advantages include the early presentation of renal injury/ dysfunction markers compared to clinical outcomes, their measurement on a continuous scale, indicating injury severity and improving statistical power for detecting differences between patient groups, and their relative site specificity, providing further insight into some AKI mechanisms. Renal injury/dysfunction markers are surrogate endpoints for more meaningful outcomes. A good surrogate endpoint both captures the effect of an intervention and predicts the ultimate clinical outcome. Current renal injury/dysfunction markers are fair surrogates. Creatinine criteria for AKI diagnosis and kidney damage biomarkers do predict short- and long-term morbidity but should be evaluated in the context of more important permanent outcomes.

New-onset CKD (stage $3 \mathrm{CKD}$, defined as eGFR $<60$ $\mathrm{ml} / \mathrm{min} / 1.73 \mathrm{~m}^{2}$ ), worsened CKD (from stage 3 or 4 to stage 4 or 5), renal replacement therapy, and death compromise hard clinical outcomes that are appropriate as primary endpoints for AKI clinical trials. The advantage of these outcomes is that they are easily measured, represent distinct events with little opportunity for misidentification, and clearly relate to long-term impairment in health and well-being of patients. The disadvantage is that they are less common than renal injury/dysfunction endpoints and surrogate outcomes and they do not provide a good opportunity to treat patients early in the course of injury.

With regard to AKI severity, use of advanced RIFLE or AKIN stage 2 or 3 AKI criteria provides a more specific AKI endpoint than less severe AKI diagnostic criteria, and therefore they may be a more appropriate endpoint when assessing the effect of treatment on AKI. This is true both for prophylactic AKI studies, such as those scheduled for cardiac surgery or intravascular iodinated radiocontrast, and particularly for treatment or rescue AKI studies, such as those administered to hospitalized patients following induction of AKI. On the other hand, less severe injury, such as stage $1 \mathrm{AKIN}$ or KDIGO, is more sensitive to AKI and will provide increased statistical power but may diag- nose AKI without definitive renal injury. This statistical advantage is important in trials of AKI prophylactic therapies when limitations of cost and recruitment feasibility demand a relatively high AKI incidence. For example, a therapy that reduces the relative risk of stage 1 AKIN AKI by $50 \%$ will require approximately 120 study subjects ( 60 in the treatment group and 60 in the control group) if the baseline risk of stage $1 \mathrm{AKIN} \mathrm{AKI} \mathrm{is} 50 \%$. A therapy that reduces the relative risk of stage 2 AKIN AKI by $50 \%$ will require approximately 560 study subjects ( 280 in the treatment group and 280 in the control group) if the baseline risk of stage 2 AKIN AKI is $15 \%$. A more sensitive criterion for AKI, such as stage I AKIN, will unfortunately increase the incidence of false-positive results and therefore attenuate the association of AKI with more important subsequent outcomes, such as worsened CKD and death.

Phase I and II clinical trials should use continuous markers of renal injury/dysfunction rather than 'hard' clinical outcomes in order to generate meaningful data with limited subject exposure to untested treatments. By doing so, investigators may assess safety without requiring large sample sizes, demonstrate treatment effect of an unknown therapeutic, and power subsequent studies. Phase III trials should incorporate consensus AKI guidelines and measure more important subsequent clinical outcomes. Secondary endpoints of phase III clinical trials could include other renal measurements not included as a primary endpoint or extrarenal endpoints often associated with AKI that increase patient morbidity, such as arrhythmia, myocardial infarction, infection, or delirium.

\section{Building Consensus for a Universal Renal Outcome of AKI}

To link AKI with persistent morbidity, the community must establish a universally accepted and employed common indicator of the chronic renal insufficiency status after AKI. Thus, investigators may best study the effect of various renal therapies on ultimate patient wellness. This outcome must be easily and objectively measured, subject to little confounding, indicative of major morbidity, and easily applied in a consistent manner to all patient populations. To assess data across clinical studies and across patient populations, analysis of outcome should be limited to a specific interval following AKI diagnosis, for example 30,60 , or 90 days. A new requirement for dialysis following AKI and persistent renal dysfunction (worsened CKD) following AKI each portend subsequent major morbidity and death. Both are easily measured using common procedural and
Billings IV/Shaw 
billing coding (dialysis) and eGFR formulae deduced from demographics and serum creatinine concentrations (persistent renal dysfunction defined as an increase in CKD stage, defined by eGFR). Mortality is the ultimate major morbid outcome following AKI and may occur prior to the initiation of dialysis or before CKD can be assessed at specific intervals. The composite outcome of death, new dialysis, and worsened renal function, which was defined as a $25 \%$ or greater decline in eGFR, constitutes the major adverse kidney event (MAKE) outcome. MAKE30 is assessed 30 days following AKI diagnosis, and MAKE60 and MAKE90, 60 and 90 days after AKI diagnosis, respectively. MAKE90 may be the most appropriate endpoint because that is typically the time when CKD is diagnosed after AKI. Employing a renal composite endpoint captures a greater percentage of patients with a meaningful poor outcome, obviates the limitation of competing risks associated with single outcomes (for example mortality prevents the development of persistent renal dysfunction), and increases the event rate for the assessment of therapies. Absence of the MAKE endpoint is also an assessment of renal disability-free survival, an important goal for all patients, providers, and hospitals. MAKE has an incidence of approximately $30 \%$ in most clinically enriched AKI risk populations, and it is important for all stakeholders because it is composed of clinical events and not simply process-of-care measures.

\section{Final Recommendations}

$\mathrm{AKI}$ is a devastating disease, but CKD is worse. The association between AKI and subsequent CKD and the imperfection of current AKI diagnostic criteria and renal damage biomarkers beseech us to include markers of hard clinical outcomes in trials of AKI. Thus, we will solidify the importance of AKI events on patient health and better understand the effects of AKI treatments. The MAKE90 composite endpoint includes persistent renal dysfunction 90 days following AKI, new-onset need for renal replacement therapy, and death, and is therefore a suitable endpoint candidate for this task.

\section{Acknowledgment}

F.T.B. was supported by the United States National Institutes of Health (K23GM102676).

\section{Disclosure Statement}

F.T.B. has no competing financial interests. A.D.S. is a consultant for Astute Medical, Baxter Healthcare, NxStage Medical, Covidien Inc., Thrasos Inc., and FAST Biomedical.

\section{References}

$\rightarrow 1$ Cooper WA, O'Brien SM, Thourani VH, Guyton RA, Bridges CR, Szczech LA, Petersen $\mathrm{R}$, Peterson ED: Impact of renal dysfunction on outcomes of coronary artery bypass surgery: results from the Society of Thoracic Surgeons National Adult Cardiac Database. Circulation 2006;113:1063-1070.

-2 Lassnigg A, Schmidlin D, Mouhieddine M, Bachmann LM, Druml W, Bauer P, Hiesmayr M: Minimal changes of serum creatinine predict prognosis in patients after cardiothoracic surgery: a prospective cohort study. J Am Soc Nephrol 2004;15:1597-1605.

-3 Chertow GM, Burdick E, Honour M, Bonventre JV, Bates DW: Acute kidney injury, mortality, length of stay, and costs in hospitalized patients. J Am Soc Nephrol 2005;16:33653370 .
4 Mehta RL, Chertow GM: Acute renal failure definitions and classification: time for change? J Am Soc Nephrol 2003;14:21782187.

5 Bihorac A, Yavas S, Subbiah S, Hobson CE, Schold JD, Gabrielli A, Layon AJ, Segal MS: Long-term risk of mortality and acute kidney injury during hospitalization after major surgery. Ann Surg 2009;249:851-858.

6 6 Bdash JR, Ishani A: Acute kidney injury and chronic kidney disease: a work in progress. Clin J Am Soc Nephrol 2011;6:2555-2557.

-7 Baigent C, Burbury K, Wheeler D: Premature cardiovascular disease in chronic renal failure. Lancet 2000;356:147-152.

-8 Devarajan P: Review: neutrophil gelatinaseassociated lipocalin: a troponin-like biomarker for human acute kidney injury. Nephrology (Carlton) 2010;15:419-428.

9 Kashani K, Al-Khafaji A, Ardiles T, et al: Discovery and validation of cell cycle arrest biomarkers in human acute kidney injury. Crit Care 2013;17:R25.
10 Bellomo R, Ronco C, Kellum JA, Mehta RL, Palevsky P: Acute renal failure - definition, outcome measures, animal models, fluid therapy and information technology needs: the Second International Consensus Conference of the Acute Dialysis Quality Initiative (ADQI) Group. Crit Care 2004;8:R204-R212.

11 Mehta RL, Kellum JA, Shah SV, Molitoris BA Ronco C, Warnock DG, Levin A; Acute Kidney Injury Network: Acute Kidney Injury Network: report of an initiative to improve outcomes in acute kidney injury. Crit Care 2004;11:R31.

12 KDIGO AKI Work Group: KDIGO clinical practice guideline for acute kidney injury. Kidney Int Suppl 2012;2:1-138. 